\title{
Assessment of Available Transfer Capability Incorporating Probabilistic Distribution of Load Using Interval Arithmetic Method
}

\author{
Prabha Umapathy, Member, IACSIT, C.Venkataseshaiah and M.Senthil Arumugam
}

\begin{abstract}
Following the deregulation of electric power utilities around the globe, it has been widely acknowledged that quantifying the power transfer capability is of increasing importance in the operation and planning of interconnected power systems. This paper reports on the application of probabilistic distribution based interval arithmetic method to estimate the Available Transfer Capability (ATC) in a power network in terms of confidence intervals. Interval arithmetic method enables incorporation of the uncertainty in the input parameters and provides strict bounds for the solution. In the present work, the variation of the real power load is represented as a Gaussian distribution function. The proposed method is tested and validated on IEEE 14 bus test system.
\end{abstract}

Index Terms-Available Transfer Capability, Interval Arithmetic, power system planning, Gaussian distribution

\section{INTRODUCTION}

Ever increasing demand for reliable and economical supply of power, led to the evolution of large interconnected power systems. As large interconnected facilities are developed, a need for trade-off between capacity and reliability arises. Deregulation of the power industry has paved a path for the re-birth of distributed energy resources and continually emerging new and difficult issues of concern in the operation of power systems. One of the emerging issues is to assess the Available Transfer Capability (ATC) in a power system.

According to NERC definition [1], ATC is a measure of the transfer capability remaining in the physical transmission network for future commercial activity over and above already committed uses. ATC can be mathematically defined as the total transfer capability (TTC) less the transmission reliability margin (TRM), less the sum of existing commitments and the capacity benefit margin (CBM), i.e., if there are no existing commitments, ATC can be expressed as ATC $=$ TTC-TRM-CBM. TRM accounts for the inherent uncertainty in the system conditions and the need for operating flexibility to ensure reliable system operation as system conditions change. CBM is the transfer capability reserved by load serving entities to ensure access to generation from the interconnected system to meet the

The authors are with the Faculty of Engineering and Technology, Multimedia University, Melaka 75450, Malaysia (e-mail: prabha.umapathy@mmu.edu.my). generation reliability requirements.

The ATC assessment involves the determination of the largest additional amount of power above some base case value that can be transferred in a prescribed manner between two sets of buses or between two areas. There are various mathematical models and algorithms which are used to compute TTC or TTC minus the base case flow [2-4]. The currently used methods can be divided into three types, namely Continuation Power Flow (CPF) method, Repeated Power Flow (RPF) method and Security Constrained Optimal Power Flow (SCOPF) method.

$\mathrm{CPF}$ is a general method for finding the maximum value of a scalar parameter in a linear function of changes in injections at a set of buses in a power flow problem. In principle, CPF increases the loading factor in discrete steps and solves the resulting power flow problem at each step. However, since CPF ignores the optimal distribution of the generation and the loading together with the system reactive power, it can give conservative transfer capability results. The $\mathrm{CPF}$, in spite of its popularity has the disadvantage of its complexity. The Repeated Power Flow (RPF) method possesses several advantages which include the ease of implementation and less time to converge [5]. SCOPF methods maximize the transfer capability between two control areas assuming all OPF-optimized parameters can be centrally dispatched. All of the above methods consider fixed input parameters (load demand and line parameters) to find the solution. These parameters are considered to be constant and deterministic. But in reality, the loads are uncertain and vary over a range.

Probabilistic or stochastic load flow methods are an approach to accommodate and model the random nature of the operational load. Quantitatively, uncertainties can be mathematically modeled with deterministic parameters as probabilistic distribution function [6,7]. In [6], the uncertainties in the load are taken into account by using Gram Charlier series considering 10\% variation in the load for calculating the probability density function. Also it is suggested that it could be beneficial for the power industry, if confidence interval calculations are made available in this application. The interval arithmetic method has been applied for power flow analysis of transmission networks in [8-10].Interval Arithmetic has been applied in the assessment of ATC by considering the uncertainties in the load and line parameters [11]. The present work proposes the use of Interval Arithmetic (IA) method for the assessment of ATC with confidence intervals, incorporating probabilistic distribution of load. In Section II, the optimal power flow 
problem is formulated and discussed. In Section III, the basic concepts of IA are explained. Section IV presents the implementation of the proposed algorithm used in the present work. Section V provides the details of the test system on which the proposed algorithm is tested and the results are presented for the same. Finally concluding remarks appear on Section VI.

\section{General Procedure And Problem Formulation}

According to NERC's definition [1], many terms have been proposed to provide additional clarifications in the calculation of ATC. They are TTC, ATC, TRM and CBM. Their definitions are as follows.

ATC is a measure of the transfer capability remaining in the physical transmission network for further commercial activity over and above already committed uses.

TTC is defined as the amount of electric power that can be transferred over the interconnected transmission network in a reliable manner while meeting all of a set of defined pre and post contingency system condition

TRM is defined as the amount of transmission transfer capability necessary to ensure that the interconnected transmission network is secure under a reasonable range of uncertainties in the system condition

CBM is defined as the amount of transmission transfer capability reserved by load serving entities to ensure access to generation from interconnected systems to meet generation reliability requirements

Determination of TTC is the key component in ATC calculation. The objective is to estimate the available transfer capability between the sending area and receiving area through the tie-lines connecting the two areas. The constraints are voltage magnitudes, generation limits and power flow limits in the lines. The mathematical formulation of TTC using Repeated Power Flow method can be expressed as follows. Equation (1) corresponds to the objective function which maximizes the active power increment of load bus and (2) corresponds to power flow equations. Equations (3), (4) and (5) give the expressions for lower and upper limits of different inequality constraints.

Objective Function

$$
\operatorname{Max}\left(\sum_{i \in S_{D}} \Delta P_{D i}\right)
$$

Subject to

Equality Constraints

$$
\begin{aligned}
& P_{G i}-P_{D i}-\sum_{j=1}^{n}\left|V_{i} \| V_{j}\right|\left(G_{i j} \cos \delta_{i j}+B_{i j} \sin \delta_{i j}\right)=0 \\
& Q_{G i}-Q_{D i}-\sum_{j=1}^{n}\left|V_{i} \| V_{j}\right|\left(G_{i j} \cos \delta_{i j}+B_{i j} \sin \delta_{i j}\right)=0
\end{aligned}
$$

Inequality Constraints

Generator output limits

$$
\begin{aligned}
& \left|P_{G i}\right|_{\min } \leq\left|P_{G i}\right| \leq\left|P_{G i}\right|_{\max } \\
& \left|Q_{G i}\right|_{\min } \leq\left|Q_{G i}\right| \leq\left|Q_{G i}\right|_{\max }
\end{aligned}
$$

Nodal voltage limits

$$
\left|V_{i}\right|_{\min } \leq\left|V_{i}\right| \leq\left|V_{i}\right|_{\max }
$$

Line thermal limits

$$
\left|P_{i j}\right|_{\min } \leq\left|P_{i j}\right| \leq\left|P_{i j}\right|_{\max }
$$

where,

$\begin{array}{ll}\Delta P_{D i} & \text { active power increment of load bus } \\ P_{G i}, Q_{G i} & \text { real and reactive power generation at bus } \mathrm{i} \\ P_{D i}, Q_{D i} & \text { real and reactive load demand at bus } \mathrm{i} \\ \mathrm{n} & \text { bus number of the system } \\ V_{i,} V_{j} & \text { voltage magnitude at bus } \mathrm{i}, \mathrm{j} \\ P_{i j} & \text { line limit }\end{array}$

\section{INTERVAL ARITHMETIC METHOD}

Interval Arithmetic is a powerful tool to determine the effects of uncertain data. It can deal with numbers that vary within a range. Interval Arithmetic can also consider in an automatic manner, the round off error accumulation during numerical computations. The basic concepts of interval arithmetic are discussed as follows.

An interval number $X=\left[x_{1}, x_{2}\right]$ is the set of real numbers $x$ such that $x_{1} \leq x \leq x_{2}$. Here $x_{1}$ and $x_{2}$ are known as the lower limit and upper limit of the interval number, respectively. Let $X=\left[x_{1}, x_{2}\right]$ and $Y=\left[y_{1}, y_{2}\right]$ be the two interval numbers. The basic arithmetic operations addition, subtraction, multiplication and division of these two interval numbers are defined in Eqns. (6)-(9) as below [12].

$$
\begin{gathered}
X+Y=\left[x_{1}+y_{1}, x_{2}+y_{2}\right] \\
X-Y=\left[x_{1}-y_{1}, x_{2}-y_{2}\right] \\
X * Y=\left[\min \left(x_{1} * y_{1}, x_{1} * y_{2}, x_{2} * y_{1}, x_{2} * y_{2}\right),\right. \\
\left.\max \left(x_{1} * y_{1}, x_{1} * y_{2}, x_{2} * y_{1}, x_{2} * y_{2}\right)\right] \\
X \div Y=X^{*} Y^{-1}
\end{gathered}
$$

However, for the purpose of power flow analysis, calculations are based on complex number rather than real numbers. The basic relations involving complex interval numbers are described as follows. In general, any complex number $\mathrm{Z}=\mathrm{X}+\mathrm{iY}$, where $\mathrm{i}$ is the complex operator, is said to be a complex interval number if both its real and imaginary part, $\mathrm{X}$ and $\mathrm{Y}$ respectively are interval numbers. Therefore $\mathrm{X}$ can be represented as $X=\left[x_{1}, x_{2}\right]$ and $\mathrm{Y}$ can be represented as $Y=\left[y_{1}, y_{2}\right]$.

Let $\mathrm{Z1}=\mathrm{A}_{1}+\mathrm{iB}_{1}$ and $\mathrm{Z}_{2}=\mathrm{A}_{2}+\mathrm{iB}_{2}$ be the two complex 
interval numbers. The addition, subtraction, multiplication and division of these two complex numbers are defined in Eqns.(10)-(13).

$$
\begin{aligned}
& Z_{1}+Z_{2}=\left(A_{1}+A_{2}\right)+i\left(B_{1}+B_{2}\right) \\
& Z_{1}-Z_{2}=\left(A_{1}-A_{2}\right)+i\left(B_{1}-B_{2}\right) \\
& Z_{1} * Z_{2}=\left(A_{1} * A_{2}-B_{1} * B_{2}\right)+i\left(A_{1} * B_{2}-A_{2} * B_{1}\right) \\
& Z_{1} \div Z_{2}=C+i D
\end{aligned}
$$

where,

$$
\mathrm{C}=\left(\mathrm{A}_{1} * \mathrm{~A}_{2}+\mathrm{B}_{1} * \mathrm{~B}_{2}\right) \div\left(\mathrm{A}_{2}^{2}+\mathrm{B}_{2}^{2}\right) \text { and }
$$$$
\mathrm{D}=\left(\mathrm{A}_{2} * \mathrm{~B}_{1}+\mathrm{A}_{1} * \mathrm{~B}_{2}\right) \div\left(\mathrm{A}_{2}^{2}+\mathrm{B}_{2}^{2}\right)
$$

\section{IMPLEMENTATION}

\section{A. Interval Power Flow Analysis and Load Variation Modelling}

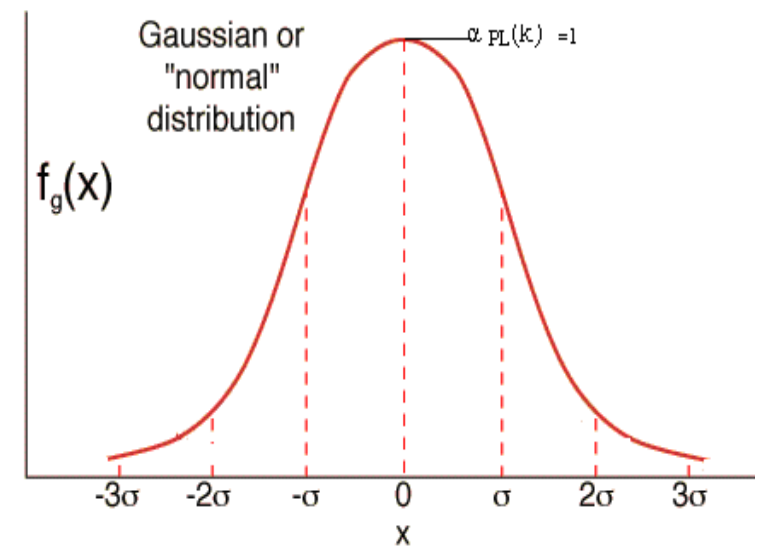

Fig.1. Gaussian distribution of load demand

Figure 1 shows the characteristic curve and it can be seen that the mean value of normalized real load is 1.0 for the degree of belongingness 1.0. The basic power flow analysis method used in this work is fast decoupled load flow algorithm. However, to account for the uncertainty of the load demands, the real power loads are treated as interval numbers rather than fixed numbers. Consequently, the complex arithmetic has been replaced by complex interval arithmetic in this proposed technique. Equation (14) corresponds to the real power load variation as per Gaussian distribution,

$$
A(y)=\frac{1}{\sigma \sqrt{2 \pi}} e \frac{-1}{2}\left(\frac{y-\mu}{\sigma}\right)^{2}
$$

where, $\pi$ is constant, $\sigma$ is the standard deviation and $\mu$ is the mean value. $y$ is the normalized real and reactive load at the $\mathrm{i}$ th bus. Fig.1. shows the characteristic curve

Let the degree of belongingness for the real and reactive load at all the buses be represented by $\alpha_{\mathrm{PL}}(\mathrm{k})$ and $\alpha_{\mathrm{QL}}(\mathrm{k})$, where $\mathrm{k}$ is the number indicating the degree of belongingness The lower and upper limit of the real power load at $i$ th bus is derived as shown in equations (15) and (16).

$$
\begin{aligned}
& P L_{l}(i)=P \operatorname{Lr}(i)\left[1-\delta_{k}\right] \\
& P L_{u}(i)=P \operatorname{Lr}(i)\left[1+\delta_{k}\right]
\end{aligned}
$$

where,

$$
\delta_{k}=\left[-\ln \alpha_{P L}(k) / \pi\right]^{1 / 2}
$$

The uncertainty due to varying load demands on each bus is addressed by assuming the random variations in the real power load as per Gaussian distribution. The reactive loads are considered constant. The load parameters, instead of assuming any value with in a predetermined closed bounded interval, varies according to Gaussian probability distribution.

\section{B. Proposed ATC Algorithm}

Step 1: Read system data

Step 2: Select the source area and sink area

Step 3: Choose the degree of belongingness $\alpha_{\mathrm{PL}}(\mathrm{k})$ for which ATC is to be calculated.( In the present work results for four different values are reported, i.e., for $0.25,0.5,0.75$ and 1)

Step 4: Using equations (15) and (16), the bounded interval of the real power load is calculated

Step 5: Establish and solve the base case power flow (no transfer). The mathematical computations in the load flow algorithm are performed using interval arithmetic.

Step 6: Use Repeated Power Flow to make a step increase in transfer power

Step 7: Establish and solve the power flow problem

Step 8: Check the obtained solution for any limit violation

Step 9: If there is any violation, decrease the transfer power by the necessary minimum amount to eliminate violation and then go to step 7 .

Step 10: Obtain the power flow solution in terms of upper limit and lower limit.

Step 11: Compute TTC and ATC in terms of upper limit and lower limit

Step 12: Print results

\section{RESULTS}

\section{A. Test System}

The IEEE 14 bus test system shown in Fig.2. is to implement the proposed algorithm. It is assumed that the system is operating under normal conditions, and the real power load is varying as per Gaussian distribution as shown in Table I for four different values of $\alpha_{\mathrm{PL}}(\mathrm{k})$.

\section{B. Division of Areas}

In order to study ATC, the system is divided into two areas, as shown in Table III. To evaluate the available transfer capability from area 1 to area 2, MATLAB simulation has been carried out by increasing the loads in area 2. The loads are increased simultaneously, the power factor remaining constant.

The proposed method provides the information in terms of 
bounded intervals for the available transfer capability. Apart

from ATC, it also enables the evaluation of bounds in terms of interval parameters for the bus voltages, real and reactive power in the branches and real and reactive power losses in the network. Figure 3 presents the graph for voltage magnitude of the load buses for the various degree of belongingness $\alpha_{\mathrm{PL}}(\mathrm{k})=1.0,0.75,0.25$.

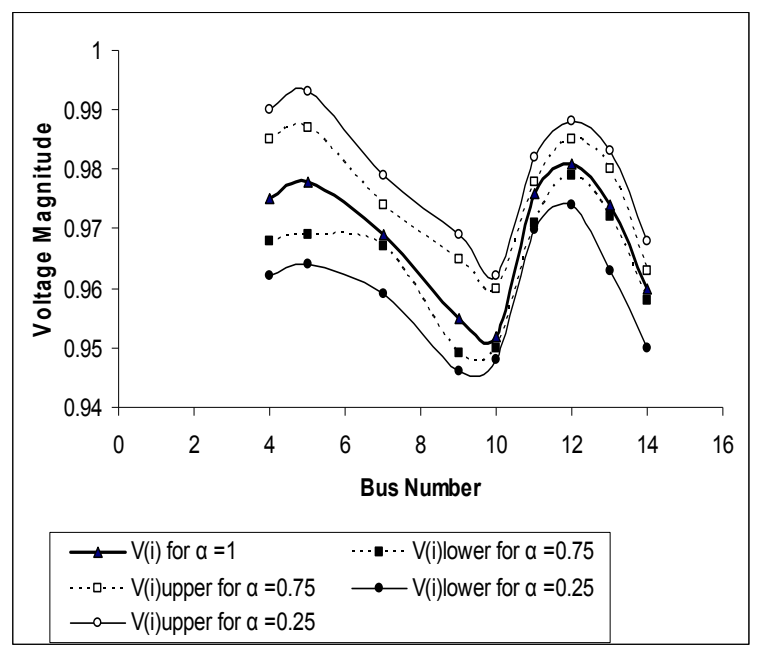

Fig.3.Voltage magnitude for various degree of belongingness

It is observed that the system voltage with nominal value $\left(\alpha_{\mathrm{PL}}(\mathrm{k})=1.0\right)$ always falls within the bounded intervals obtained for different degrees of belongingness. These bounded interval values provide the necessary information to assess the robustness of the system performance. For a specific case of $\alpha_{\mathrm{PL}}(\mathrm{k})=0.75$, the available transfer capability between area 1 and area 2 , which are connected by the tie lines between buses 5 and 6 and buses 4 and 7 is found to be 27.85 MW(lower limit) and 52.07 MW (Upper limit). The voltage collapse is observed at buses 13 and 14. Also, the stability limit is violated in the branch connecting the buses 1 and 5 . In a similar manner, the transfer capabilities are calculated for different degree of belongingness and the various limiting factors are evaluated. The base case results are presented in Table II and the results of ATC obtained for different values of $\alpha_{\mathrm{PL}}(\mathrm{k})$ are presented in Table IV.

Figure 4 shows the available power transfer from area 1 to area 2 as a function of degree of belongingness. The shape of the curve follows the Gaussian distribution, confirming that the representation of probabilistic load variation using interval arithmetic approach gives results which closely agree with those obtained using probabilistic approach. The interval arithmetic approach has the advantage of reduced computational complexity.

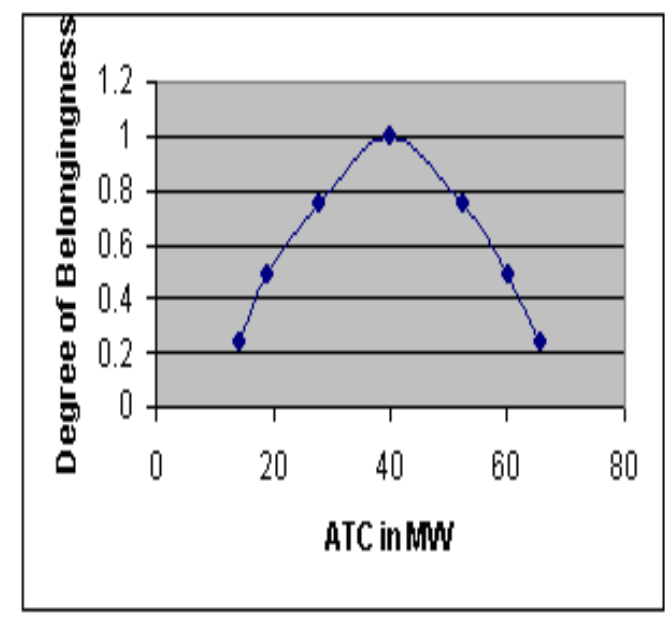

Fig.4.ATC as a function of degree of belongingness

\section{CONCLUSION}

Available transfer capability evaluation using interval arithmetic method incorporating the uncertainty in the real power load has been proposed. The probabilistic variations of load are linearized at multiple points to find bounded intervals. The proposed method provides more information about the system in quantitative terms when compared with the conventional approach which considers the load as constant. The IA approach enables the assessment of ATC in terms of confidence intervals considering the probabilistic distribution of the load variation The results obtained using IEEE 14 bus test system validates this approach

\section{REFERENCES}

[1] Available transfer capability definitions and determination, NERC, Reference document, 1996

[2] Ajjarapu.V and C.Christy, "The continuation power flow: A tool for steady state voltage stability analysis," IEEE Transactions on Power Systems, 1992, Vol.7 : 416-423.

[3] Ejebe. G.C., J.Tong, J.G.Waight, J.G.Frame, X.Wang and W.F.Tinney, "Available transfer Capability calculations, IEEE Transactions on Power Systems, 1998,Vol.13 : 1521-1527.

[4] Yan.O.U and Chanan Singh, "Assessment of available transfer capability and margins", IEEE Transactions on Power Systems, 2002, Vol.17 : 463-468.

[5] Prabha Umapathy and C.Venkataseshaiah, "A novel approach for the assessment of available transfer capability in power systems, College science in India, 2007, Vol 1:3:1-4.

[6] Gerald T.Heydt and Jonathan.W.Stahlhut, "The evaluation of stochastic available transfer capability for transmission expansion”, PSERC report, 2007.

[7] Paramasivam Venkatesh, Gnanadass Ramachandran and Narayana Prasad Padhy, "Available Transfer Capability Determination Using Power Transfer Distribution Factors", International Journal of Emerging Electric Power Systems,2004, Vol. 1 : Iss. 2, Article 1009.

[8] Zian Wang and Fernando.L.Alvarado, "Interval arithmetic in power flow analysis", Transactions on Power Systems, 1992,Vol.7 : 1341-1349.

[9] Biswarup Das, Radial distribution system power flow using interval arithmetic, Electrical power and energy systems, 2002, $24: 827-836$

[10] Chaturvedi.A., K.Prasad and R.Ranjan, "Use of interval arithmetic to incorporate the uncertainty of load demand for radial distribution system analysis", IEEE Transactions on Power delivery, 2006, Vol.21 : 1019-1021.

[11] Prabha Umapathy , C.Venkataseshaiah and M.Senthil Arumugam, "Application of Interval Arithmetic in the Evaluation of Transfer Capabilities considering the sources of uncertainty" ,International Journal-- Discrete Dynamics in Nature and Society, Vol.2009, Article ID 527385, ISSN 1026-0226. 


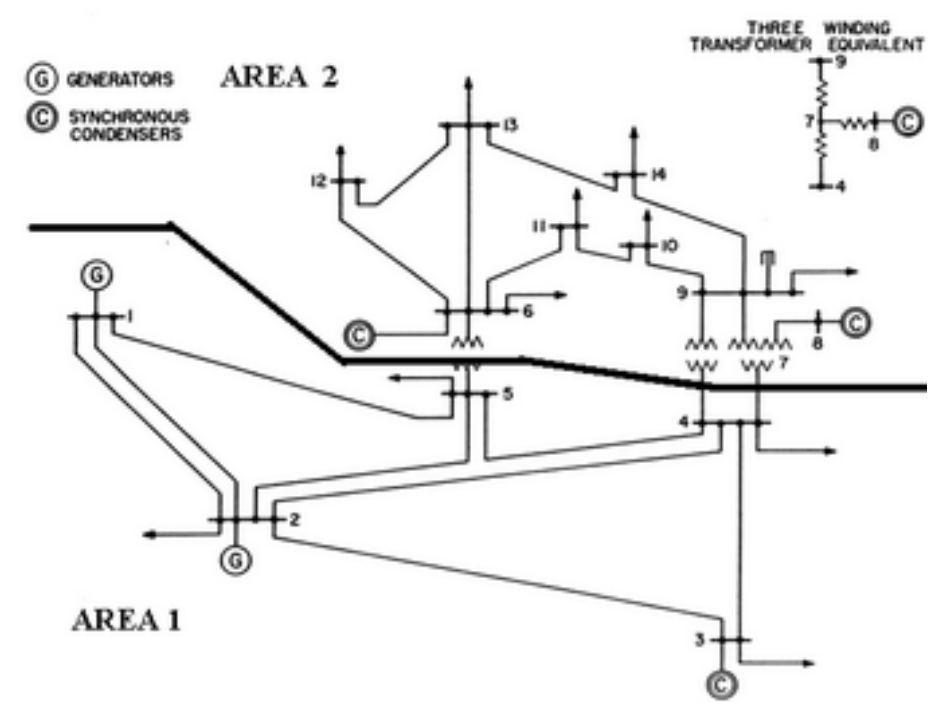

Fig.2. IEEE 14 bus test system

TABLE I : VARIATION IN REAL LOAD REPRESENTED AS GAUSSIAN DISTRIBUTION FUNCTION FOR DiFFERENT VALUES OF DEGREE OF BELONGINGNESS

\begin{tabular}{|c|c|c|c|c|c|c|c|c|}
\hline $\begin{array}{l}\text { Load } \\
\text { Bus } \\
\text { No. }\end{array}$ & $\begin{array}{l}\alpha_{\mathrm{PL}(\mathrm{k})=1} \\
\text { (Lower) }\end{array}$ & $\begin{array}{l}\alpha_{\mathrm{PL}(\mathrm{k})=1} \\
(\mathrm{Upper})\end{array}$ & $\begin{array}{l}\alpha_{\mathrm{PL}(\mathrm{k})=0.75} \\
\text { (Lower) }\end{array}$ & $\begin{array}{l}\alpha_{\mathrm{PL}(\mathrm{k})=0.75} \\
\text { (Upper) }\end{array}$ & $\begin{array}{l}\alpha_{\mathrm{PL}(\mathrm{k})=0.5} \\
\text { (Lower) }\end{array}$ & $\begin{array}{l}\alpha_{\mathrm{PL}(\mathrm{k})=0.5} \\
\text { (Upper) }\end{array}$ & $\begin{array}{l}\alpha_{\mathrm{PL}(\mathrm{k})=0.25} \\
\text { (Lower) }\end{array}$ & $\begin{array}{l}\alpha_{\mathrm{PL}(\mathrm{k})=0.25} \\
\text { (Upper) }\end{array}$ \\
\hline 2 & 21.7 & 21.7 & 15.14 & 28.27 & 11.51 & 31.89 & 7.28 & 36.11 \\
\hline 3 & 94.2 & 94.2 & 65.69 & 122.7 & 49.96 & 138.44 & 31.62 & 156.77 \\
\hline 4 & 47.8 & 47.8 & 33.34 & 62.26 & 25.35 & 70.25 & 16.05 & 79.55 \\
\hline 5 & 7.6 & 7.6 & 5.3 & 9.89 & 4.03 & 11.17 & 2.55 & 12.65 \\
\hline 6 & 11.2 & 11.2 & 7.81 & 14.59 & 5.94 & 16.46 & 3.76 & 18.64 \\
\hline 9 & 29.5 & 29.5 & 20.57 & 38.43 & 15.64 & 43.36 & 9.90 & 49.09 \\
\hline 10 & 9 & 9 & 6.28 & 11.72 & 4.77 & 13.23 & 3.02 & 14.98 \\
\hline 11 & 3.5 & 3.5 & 2.44 & 4.56 & 1.86 & 5.14 & 1.17 & 5.83 \\
\hline 12 & 6.1 & 6.1 & 4.25 & 7.95 & 3.23 & 8.97 & 2.05 & 10.15 \\
\hline 13 & 13.5 & 13.5 & 9.41 & 17.59 & 7.16 & 19.84 & 4.53 & 22.47 \\
\hline 14 & 14.9 & 14.9 & 10.4 & 19.41 & 7.9 & 21.89 & 5 & 24.8 \\
\hline
\end{tabular}

TABLE II : BASE CASE LOAD FLOW

\begin{tabular}{|c|c|c|c|c|c|c|c|}
\hline \multirow[b]{2}{*}{ Bus number } & \multirow[b]{2}{*}{ Area number } & \multirow[b]{2}{*}{ V (p.u) } & \multirow[b]{2}{*}{ Angle(deg) } & \multicolumn{2}{|c|}{ Generation } & \multicolumn{2}{|l|}{ Load } \\
\hline & & & & $\mathrm{P}$ & $\mathrm{Q}$ & $\mathrm{P}$ & $\mathrm{Q}$ \\
\hline 1 & 1 & 1 & 0 & 234.84 & 0.0 & -- & -- \\
\hline 2 & 1 & 1 & -5.9 & 40.00 & 47.38 & 21.7 & 12.7 \\
\hline 3 & 1 & 1 & -14.6 & 0.0 & 63.21 & 94.2 & 19.00 \\
\hline 4 & 1 & 0.978 & -11.6 & -- & -- & 47.8 & -3.9 \\
\hline 5 & 1 & 0.979 & -10.05 & -- & -- & 7.60 & 1.60 \\
\hline 6 & 2 & 1 & -16.65 & 0.0 & 40.53 & 11.20 & 7.50 \\
\hline 7 & 2 & 0.975 & -15.1 & -- & -- & -- & -- \\
\hline 8 & 2 & 1 & -15.1 & 0.0 & 14.02 & -- & -- \\
\hline 9 & 2 & 0.959 & -16.97 & -- & -- & 29.50 & 16.6 \\
\hline 10 & 2 & 0.958 & -17.24 & -- & -- & 9.00 & 5.80 \\
\hline 11 & 2 & 0.975 & -17.09 & -- & -- & 3.50 & 1.80 \\
\hline 12 & 2 & 0.983 & -17.64 & -- & -- & 6.10 & 1.60 \\
\hline 13 & 2 & 0.976 & -17.68 & -- & -- & 13.50 & 5.80 \\
\hline 14 & 2 & 0.947 & -18.47 & -- & -- & 14.90 & 5.0 \\
\hline \multirow[b]{2}{*}{ Bus number } & \multirow[b]{2}{*}{ Area number } & \multirow[b]{2}{*}{ V (p.u) } & \multirow[b]{2}{*}{ Angle(deg) } & \multicolumn{2}{|c|}{ Generation } & \multicolumn{2}{|l|}{ Load } \\
\hline & & & & $\mathrm{P}$ & Q & $\mathrm{P}$ & Q \\
\hline 1 & 1 & 1 & 0 & 234.84 & 0.0 & -- & -- \\
\hline 2 & 1 & 1 & -5.9 & 40.00 & 47.38 & 21.7 & 12.7 \\
\hline 3 & 1 & 1 & -14.6 & 0.0 & 63.21 & 94.2 & 19.00 \\
\hline 4 & 1 & 0.978 & -11.6 & -- & -- & 47.8 & -3.9 \\
\hline
\end{tabular}


International Journal of Computer and Electrical Engineering, Vol. 2, No. 4, August, 2010 1793-8163

\begin{tabular}{|l|l|l|l|l|l|l|l|}
5 & 1 & 0.979 & -10.05 & -- & -- & 7.60 & 1.60 \\
\hline 6 & 2 & 1 & -16.65 & 0.0 & 40.53 & 11.20 & 7.50 \\
\hline 7 & 2 & 0.975 & -15.1 & -- & -- & -- & -- \\
\hline 8 & 2 & 1 & -15.1 & 0.0 & 14.02 & -- & -- \\
\hline 9 & 2 & 0.959 & -16.97 & -- & -- & 29.50 & 16.6 \\
\hline 10 & 2 & 0.958 & -17.24 & -- & -- & 9.00 & 5.80 \\
\hline 11 & 2 & 0.975 & -17.09 & -- & -- & 3.50 & 1.80 \\
\hline 12 & 2 & 0.983 & -17.64 & -- & -- & 6.10 & 1.60 \\
\hline 13 & 2 & 0.976 & -17.68 & -- & -- & 13.50 & 5.80 \\
\hline 14 & 2 & 0.947 & -18.47 & -- & -- & 14.90 & 5.0 \\
\hline
\end{tabular}

TABLE III. DIVISION OF AREAS

\begin{tabular}{l|l}
\hline Area & Buses \\
\hline 1 & $1,2,3,4,5$ \\
\hline 2 & $6,7,8,9,10,11,12,13,14$ \\
\hline
\end{tabular}

TABLE IV. RESULTS OF ATC IN INTERVAL FORM

\begin{tabular}{ccc}
\hline & \multicolumn{2}{c}{ ATC (MW) } \\
$\alpha_{\text {PL }}(\mathrm{k})$ & Lower Limit & Upper Limit \\
& & \\
\hline & & \\
1 & 39.97 & 39.97 \\
0.75 & 27.85 & 52.07 \\
0.5 & 19.86 & 58.12 \\
0.25 & 14.30 & 65.41 \\
\end{tabular}

